\title{
Determinación del contenido de Nitratos y Nitritos en Brócoli (Brassica oleracea var. Italica) Comercializados en la Ciudad de Osorno, Chile
}

\author{
Emir Valencia ${ }^{(1)^{\star}}$, Eugenia Valenzuela(1), Roberto Quevedo ${ }^{(2)}$ y Vilma Aedo(1) \\ Universidad de Los Lagos, Departamento de Acuicultura y Recursos Agroalimentarios, (1) Laboratorio de \\ Investigación Química, Programa FITOGEN, (2) Programa Fitogen, Avenida Fuchslocher 1305, Osorno. \\ Chile. (e-mail: evalen@ulagos.cl)
}

${ }^{*}$ Autor a quien debe ser dirigida la correspondencia

Recibido Ago. 22, 2014; Aceptado Oct. 27, 2014; Versión final recibida Dic. 24, 2014

\section{Resumen}

Se analizaron los niveles de nitratos y nitritos en el brócoli crudos y cocidos, provenientes de las regiones chilenas de Coquimbo, Valparaíso y Metropolitana, que se comercializan y consumen en la ciudad sureña de Osorno en Chile. En los productos crudos (nitratos extraídos con solución extractiva de cloruro de cadmio y cloruro de bario), se encontraron niveles de nitratos entre 521 y $5638 \mathrm{mg} \mathrm{NO}_{3}^{-} / \mathrm{kg}$, mientras que en los productos cocidos (nitratos solubles en agua hervida durante 10 minutos), no se detectaron trazas de nitrito y bajos niveles de nitrato (124 a $952 \mathrm{mg} \mathrm{NO}_{3}^{-} / \mathrm{kg}$ ). De los resultados se concluye que el brócoli estudiado es de buena calidad y están bajo los valores aceptables reportados en la literatura.

Palabras clave: brócoli, Brassica oleracea var. Italica, nitratos, nitritos

\section{Determination of Nitrate and Nitrite Compounds from Broccoli (Brassica oleracea var. Italica) Commercialized in the City of Osorno, Chile}

\begin{abstract}
Levels of nitrates and nitrites in the raw and cooked broccoli, from the Chilean Regions of Coquimbo, Valparaiso and Metropolitan, which is marketed and consumed in the southern city of Osorno in Chile were analysed. In raw product (nitrate extraction procedures by means of cadmium and barium chloride solution), the nitrate levels were between 521 to $5638 \mathrm{mg} \mathrm{NO}_{3}{ }^{-} / \mathrm{kg}$, while the boiled products (soluble nitrates in boiled water for 10 minutes), no traces of nitrite were detected and low levels of nitrate (124 to $952 \mathrm{mg} \mathrm{NO}_{3}-\mathrm{kg}^{-}$) . From the results it is concluded that the broccoli samples studied are of good quality and are under acceptable values reported in the literature.
\end{abstract}

Keywords: broccoli, Brassica oleracea var. Italica, nitrates, nitrites 


\section{INTRODUCCIÓN}

Recientemente, el consumo de vegetales y hortalizas se ha incrementado alentado por el aumento de dietas vegetarianas que promueven la disminución del consumo de proteínas y grasas animales y el aumento en el aporte de fibras, minerales, vitaminas y ácidos grasos esenciales. Se observa en Chile un creciente interés por incorporar en la dieta el brócoli (Brassica oleracea var. italica) (Fundación Chile, 2013). El mayor consumo de crucíferas (col, brócoli, coliflor, entre otros) puede reducir el riesgo de cáncer colo-rectal y de tiroides (Jacoby y Keller, 2006; Bryan et al., 2012). El brócoli es originario de las costas del Mediterráneo y Asia Occidental (Grecia, Turquía, Siria), en Chile se introdujo con la colonización española extendiéndose rápidamente a lo largo del territorio, donde encontraron condiciones ambientales optimas para desarrollarse (Aljaro, 2000).

La ingesta de nitratos y nitritos en la dieta humana debería ser controlada y podría ser considerada como un factor de riesgo para la salud. Aunque los nitratos son relativamente inofensivos para los humanos, su conversión a nitritos u otros compuestos como N-nitroso pueden producir sustancias tóxicas (DomínguezGento, 1994; Merusi et al., 2010; Lammarino et al., 2013; Bryan et al., 2012). Aunque el contenido de nitratos en alimentos ha sido poco estudiado, se conoce que los niveles de nitratos en los vegetales varían dependiendo de las condiciones de desarrollo: fertilización biológica, tipos de fertilizantes nitrogenados usados, cultura hidropónica, estación de cosecha, condiciones de luz y temperatura (Huarte-Mendioca et al., 1997; Lammarino et al., 2013; Correia et al., 2010). Los vegetales aportan el $87 \%$ de los nitratos en una dieta normal, como consecuencia de las ensaladas provienen el $70 \%$ de los nitratos que son absorbidos, el resto se obtiene de las aguas y carnes (Escoin et al., 1998; NAS, 1981). El objetivo de este estudio fue determinar las diferencias significativas entre el nivel de nitratos y nitritos presentes en brócoli crudos y en brócolis cocidos, comercializados en la ciudad de Osorno, Chile.

\section{MATERIALES Y MÉTODOS}

Las muestras de brócoli correspondieron a la cosecha de otoño 2011, físicamente presentaron las mismas cualidades en cuanto al tamaño de pellas (aproximadamente de $10 \mathrm{~cm}$ de diámetro y $150 \mathrm{~g}$ de peso). Lotes correspondientes a diferentes agroindustrias (ocho) del país fueron seleccionados de acuerdo a la disponibilidad del mercado local, en la ciudad de Osorno. Las muestras fueron obtenidas aleatoriamente y analizadas por triplicado. El origen de las muestras analizadas, se presentan en la Tabla 1.

Tabla 1: Origen de las muestras. * Las muestras B5 y B8 corresponden a dos agroindustrias distintas en la misma localidad.

\begin{tabular}{|c|c|c|}
\hline Muestra & Localidad & Región chilena \\
\hline B1 & La Serena & Coquimbo \\
\hline B2 & Coquimbo & Coquimbo \\
\hline B3 & Quillota & Valparaíso \\
\hline B4 & Curacaví & Valparaíso \\
\hline B5 & Santiago & Metropolitana \\
\hline B6 & Colina & Metropolitana \\
\hline B7 & Paine & Metropolitana \\
\hline B8 & Santiago & Metropolitana \\
\hline
\end{tabular}

Las muestras fueron usadas crudas y cocidas. Para las muestras crudas, se pesaron $100 \mathrm{~g}$ de brócoli y se molieron en una licuadora marca Sindelen modelo L200 a 1000 rpm. Se agregó $100 \mathrm{~mL}$ de solución extractiva (50 g de $\mathrm{CdCl}_{2}$ y $50 \mathrm{~g}$ de $\mathrm{BaCl}_{2}$ disueltos en $1000 \mathrm{~mL}$ de agua desionizada) y $800 \mathrm{~mL}$ de agua, incluyendo el volumen de agua que aporta la muestra. La mezcla se llevó a un agitador magnético marca Heidolph por espacio de un minuto a 500 rpm, se vació en un recipiente y se dejó reposar durante una hora. Posteriormente se agregaron $100 \mathrm{~mL}$ de solución buffer (preparada disolviendo $50 \mathrm{~g}$ de $\mathrm{NH}_{4} \mathrm{Cl}$ en $500 \mathrm{~mL}$ de agua y ajustado a pH 9.6 con $\mathrm{NH}_{4} \mathrm{OH}$ ), se completó a un litro, se mezcló y se procedió a filtrar (AOAC, 1995), para obtener un líquido para análisis. 
Para la muestra cocida, se pesaron $65 \mathrm{~g}$ de brócoli los que se calentaron a $100^{\circ} \mathrm{C}$ en $500 \mathrm{~mL}$ de agua desionizada durante 10 minutos. Se filtró en caliente usando papel Whatman $\mathrm{N}^{\circ} 40$. El filtrado fue almacenado entre $4^{\circ} \mathrm{C}$ a $5^{\circ} \mathrm{C}$ por 24 horas (Huarte-Mendioca et al., 1997). El contenido de humedad se determinó por método termogravimétrico a $105^{\circ} \mathrm{C}$ (AOAC, 1995). El nivel de nitratos fue determinado por el método de reducción a nitritos, de acuerdo al método de Kramlich (Kramlich et al., 1973). Los nitritos se determinaron por espectrofotometría a $540 \mathrm{~nm}$ usando un equipo Metertek SP-850. Las muestras fueron tratadas con sulfanilamida y dihidroclorohidrato de $\mathrm{N}$ - (1 - naftil) - etilen diamina (AOAC, 1995).

Esta metodología fue validada dentro del laboratorio en base a uso de blancos (limite de detección), las curvas fueron elaboradas con material de referencia estandarizado, y se hicieron replicas de muestras obteniendo un valor menor al $5 \%$ de precisión. Para encontrar diferencias significativas entre tratamiento (cocido y crudo), se uso el Test estadístico T-Students a 5\% de nivel de significancia; mientras que para las localidades la prueba Kruskal-Wallis. La prueba de Tukey modificada (Sokal y Rohlf, 2002) fue aplicada en caso de encontrar diferencias significativas.

\section{RESULTADOS Y DISCUSIÓN}

En la Tabla 2 se presentan los resultados del contenido de humedad para el brócoli correspondiente a cada localidad de estudio. Los valores encontrados son similares a los reportados por otro autores: $85,42-91,44 \%$ (Campas-Baypoli et al., 2009) y 92,11\% ( Solano et al., 2003).

Tabla 2: Porcentajes de humedad en muestras de Brassica oleracea.

\begin{tabular}{|c|c|}
\hline Brocoli & Promedio \\
\hline B1 & $86.41 \pm 0.11$ \\
\hline B2 & $87.36 \pm 0.25$ \\
\hline B3 & $85.55 \pm 0.06$ \\
\hline B4 & $84.99 \pm 0.15$ \\
\hline B5 & $88.94 \pm 0.15$ \\
\hline B6 & $85.60 \pm 0.88$ \\
\hline B7 & $86.30 \pm 0.14$ \\
\hline B8 & $91.60 \pm 0.15$ \\
\hline
\end{tabular}

Tabla 3: Cantidad de nitratos y nitritos en muestra cocida y en muestra cruda. N.D.: No Detectado; $\bar{X}$ : promedio $\mathrm{mg} \mathrm{NO}_{3} / \mathrm{kg}$ base seca.

\begin{tabular}{|c|c|c|c|c|c|c|c|c|}
\hline & \multicolumn{4}{|c|}{ Muestras cocidas } & \multicolumn{4}{|c|}{ Muestras crudas } \\
\hline Brócoli & $\begin{array}{c}m g \mathrm{NO}_{3}{ }^{-} / \mathrm{kg} \\
\text { Base Seca }\end{array}$ & $\sigma$ & $\bar{x}$ & $\begin{array}{c}m g \mathrm{NO}_{2}-1 \mathrm{~kg} \\
\text { Base Seca }\end{array}$ & $\begin{array}{c}\mathrm{mg} \mathrm{NO}_{3}{ }^{-} / \mathrm{kg} \\
\text { Base Seca }\end{array}$ & $\sigma$ & $\bar{x}$ & $\begin{array}{c}\mathrm{mg} \mathrm{NO}_{2} / \mathrm{kg} \\
\text { Base Seca }\end{array}$ \\
\hline B1 & $\begin{array}{l}1301.4 \\
1279.4\end{array}$ & \pm 15.58 & \multirow{2}{*}{952.6} & N.D. & $\begin{array}{l}6126.6 \\
6148.7\end{array}$ & \pm 15.63 & \multirow{2}{*}{5638.2} & N.D. \\
\hline B2 & $\begin{array}{l}632.02 \\
597.50\end{array}$ & \pm 24.41 & & N.D. & $\begin{array}{l}5155.5 \\
5121.9\end{array}$ & \pm 23.76 & & N.D. \\
\hline B3 & $\begin{array}{l}154.08 \\
152.58\end{array}$ & \pm 1.06 & \multirow{2}{*}{177.9} & N.D. & $\begin{array}{l}518.48 \\
517.57\end{array}$ & \pm 0.64 & \multirow{2}{*}{521.4} & $<1$ \\
\hline B4 & $\begin{array}{l}201.32 \\
203.53\end{array}$ & \pm 1.56 & & N.D. & $\begin{array}{l}522.63 \\
526.82\end{array}$ & \pm 2.96 & & $<1$ \\
\hline B5 & $\begin{array}{l}302.78 \\
301.80\end{array}$ & \pm 0.69 & \multirow{4}{*}{123.9} & N.D. & $\begin{array}{l}1962.7 \\
1962.7\end{array}$ & \pm 0.00 & \multirow{4}{*}{683.8} & N.D. \\
\hline B6 & $\begin{array}{l}170.97 \\
170.97\end{array}$ & \pm 0.00 & & N.D. & $\begin{array}{l}577.53 \\
575.94\end{array}$ & \pm 1.20 & & N.D. \\
\hline B7 & $\begin{array}{l}3.01 \\
3.01\end{array}$ & \pm 0.00 & & N.D. & $\begin{array}{l}55.03 \\
53.06\end{array}$ & \pm 1.39 & & N.D. \\
\hline B8 & $\begin{array}{l}18.93 \\
20.49\end{array}$ & \pm 1.10 & & N.D. & $\begin{array}{l}143.65 \\
139.87\end{array}$ & \pm 2.67 & & N.D. \\
\hline
\end{tabular}


En la Tabla 3 se presentan las concentraciones de nitratos y nitritos presentes tanto en muestras cocidas como en muestras crudas en base seca. Se aprecia que no existe evidencia de nitrito en las muestras, pero sí existen diferencias en las concentraciones de nitrato. De acuerdo al análisis estadístico Tstudent (Tabla 3) no hubo diferencia significativa en la concentración de nitratos entre muestras de brócoli cocidas y crudas (5\% de nivel de significancia). Sin embargo, si hubo diferencias significativas entre las muestras cocidas y crudas $(t=3,169$; $\mathrm{p}=0,006)\left(\mathrm{U}_{\mathrm{M}-\mathrm{W}}=72 ; \mathrm{p}=0.03\right)$. Con respecto a la concentración de nitratos en las muestras de brócoli cocidos (entre localidades) hubo diferencias significativa (Kruskal-Wallis $=8,7610 ; p=0,0125$ ). De igual forma la concentración de nitratos en producto crudo (entre localidades) presenta diferencia significativamente (KruskalWallis = 8,4830; $p=0,0144$ ). De acuerdo a la prueba de Tukey modificada, se determinó que la concentración de nitratos en brócoli cocido proveniente de la región de Coquimbo fue mayor que las detectadas en la región de Valparaíso y Metropolitana.

Referido a los resultados obtenidos el contenido de nitrito y nitrato para brócoli fresco Hsu et al., 2009 , reportaron valores de $0 \mathrm{mg} / \mathrm{Kg}$ y 236,2 mg/kg; Correia et al., 2010, valores entre 0,8 y $30 \mathrm{mg} / \mathrm{kg}$ y 41 y 939 $\mathrm{mg} / \mathrm{kg}$ respectivamente, mientras que para Leszczyríska et al., 2009, los valores fluctuaron entre 1,90 y $277,7 \mathrm{mg} / \mathrm{kg}$. En nuestra investigación no se detectaron trazas de nitrito, sólo bajos niveles de nitrato en los productos cocidos (124 - $952 \mathrm{mg} \mathrm{NO}_{3}^{-} / \mathrm{kg}$ ), no así en los productos crudos (521 - $\left.5638 \mathrm{mg} \mathrm{NO}_{3^{-}} / \mathrm{kg}\right)$. La OMS y la $\mathrm{FAO}$ indican $3,7 \mathrm{mg} \mathrm{NO}{ }_{3} / \mathrm{kg}$ peso y $0,133 \mathrm{mg} \mathrm{NO}_{2} / \mathrm{kg}$ peso, como dosis diaria admisible en adultos (La Malfa, 1988); Bryan et al., 2012 reportan que el consumo de nitrato endógeno desde una dieta puede llegar a ser entre 50 a 220 mg/día; y para el nitrito entre 0 a 0,7 mg/día. De acuerdo a los resultados obtenidos en el análisis de las muestras, se concluye que estas son de buena calidad.

\section{CONCLUSIONES}

Del estudio realizado, de los resultados y de su discusión se pueden extraer cuatro conclusiones principales:

1) Se encontró sólo nitratos y no se detectaron trazas de nitrito;

2) el lugar de procedencia de las muestras afecta los niveles de nitrato tanto para las muestras crudas como cocidas, encontrándose que las muestras provenientes de la región de Coquimbo presentan niveles mayores comparados con las provenientes de las regiones de Valparaíso y Metropolitana, que estadísticamente no difieren;

3) la diferencia existente entre las muestras de distinta procedencia podría estar determinada por las condiciones de cultivo, por la fertilización efectuada, por el clima de la zona, condiciones de irrigación y por las condiciones del suelo; y

4) entre muestras provenientes de la misma Región, no se observaron grandes diferencias, salvo en dos muestras provenientes de la Región Metropolitana, cuya diferencia pudiera deberse al manejo del cultivo.

\section{AGRADECIMIENTOS}

Los autores agradecen el apoyo y colaboración de la Dirección de Investigación de la Universidad de Los Lagos.

\section{REFERENCIAS}

AOAC, Official Methods of Analysis, $16^{\text {th }}$ Ed International, Washington DC (1995)

Aljaro, A., "Cultivos de brasicas. Repollo, coliflor, brocoli, repollito de bruselas", Tierra Adentro. №34, 12-14 (2000)

Bryan, N., D. Alexander, J. Coughlin. A. Milkowski y P. Boffetta, "Ingested nitrate and nitrite and stomach cancer risk: An updated review". Food and Chemical Toxicology, 50, 3646-3665 (2012)

Campas-Baypoli, O., C. Bueno-Solano, D. M. Martínez-Ibarra, F. Camacho-Gil, A.G. Villa-Lerma, J.R. Rodríguez-Núñez, J. López-Cervantes y D.I. Sánchez-Machado, "Contenido de sulforafano (1-isotiocianato4-(metilsulfinil)-butano) en vegetales crucíferos". Archivos Latinoamericanos de Nutrición, Vol. 59 (1), 95-100 (2009)

Correia, M., A. Barroso, M.F. Barroso, D. Soares, M.B.P.P. Oliveira y C. Delerue-Matos, "Contribution of different vegetable types to exogenous nitrate and nitrite exposure". Food Chemistry, 120, 960-966 (2010) 
Domínguez-Gento, P. y A. Domínguez-Gento, "Nitratos en lechugas procedentes de cultivos convencionales y ecológicos en la provincia de Valencia". I Congreso de la Sociedad Española de Agricultura Ecológica, Toledo, España, 523-527 (1994)

Escoín-Peña, M.C., M.A.Cortés Ibañez, A. Arranz Santamaría y L.C. Rubio, "Contenido de nitrato en lechugas y espinacas frescas". Alimentaria 298: 37-41 (1998)

Fundación Chile, "Estudio Chile Saludable: oportunidades y desafíos de innovación". Volumen II. Pág.20, Mayo 2013. Disponible en : http://www.fundacionchile.com/archivos/ESTUDIO-CHILE-SALUDABLEVOLUMEN-II.pdf. Acceso: 3 de Noviembre (2014)

Huarte-Mendicoa, J.C., I. Astiasarán y J. Bello, "Nítrate and nitrite levels in fresh and frozen broccoli. Effect of freezing and cooking", Food Chemistry, 58(1-2), 39-42 (1997)

Hsu, J., J. Arcot, N.A. Lee, "Nitrate and nitrite quantification from cured meat and vegetables and their estimated dietary intake in Australians". Food Chemistry, 115, 334-339 (2009)

Jacoby E. y I. Keller, "La promoción del consumo de frutas y verduras en América Latina: buena oportunidad de acción intersectorial por una alimentación saludable". Rev Chil Nutr. 33, 226-231, Suplemento №1, (2006)

Kramlich, W.E., A.M. Pearson y F.W. Taüber, "Processed Meat” AVI Publishing Co., Westport (1973)

La Malfa, G., "Nuovi orientamenti dei consumi e delle produzioni alimentari. La qualitá degli ortaggi destinati al consumo allo stato fresco". Cons. Nazi. delle Ricerche. 69, Torino. Italia (1988)

Leszczyńska, T., A. Filipiak-Florkiewicz, E. Cieślik, E. Sikora y P. M. Pisulewski, "Effects of some processing methods on nitrate and nitrite changes in cruciferous vegetables". Journal of Food Composition and Analysis, 22, 315-321 (2009)

NAS (National Academy of Science), "The health effects of nitrate, nitrite and N-nitroso compounds". Assembly of Life Sciences. National Academy Press, Washington D.C. (1981)

Maldonado, R. J. y E. Pacheco-Delahaye, "Curvas de deshidratación del brócoli (Brassica oleraceae $L$ var. Italica Plenk) y coliflor (Brassica oleraceae L var. Botrytis L)". Revista Facultad de Agronomía (LUZ) 20, 306 -319 (2003)

Meah, M.N., N. Harrison y A. Davies, "Nitrate and nitrite in foods and the diet", Food Additives \& Contaminants: Part A: Chemistry, Analysis, Control, Exposure \& Risk Assessment, 11, º 4, 519-532 (1994)

Merusi, C., C. Corradini, A. Cavaza, Ch. Borromei y P. Salvadeo, "Determination of nitrates, nitrites and oxalates in food products by capillary electrophoresis with pH-dependent electroosmotic flow reversal". Food Chemistry, 120, 615-620 (2010)

Sokal, R. y J. Rohlf, "Serie de Biología Fundamental. Introducción a la Bioestadística",Reverté, México (2002) 
